\title{
Configuration of DNA strands and mechanism of strand exchange in the Hin invertasome as revealed by analysis of recombinant knots
}

\author{
Karen A. Heichman, Ivan P.G. Moskowitz, ${ }^{1}$ and Reid C. Johnson ${ }^{2}$ \\ Department of Biological Chemistry, University of California at Los Angeles School of Medicine and Molecular Biology \\ Institute, University of California, Los Angeles, California 90024 USA
}

The Hin recombinase of Salmonella normally catalyzes a site-specific DNA inversion reaction that is very efficient when the Fis protein and a recombinational enhancer sequence are present. The mechanism of this recombination reaction has been investigated by analyzing the formation and structure of knots generated in different plasmid substrates in vitro. Hin seldom knots the wild-type substrate under standard recombination conditions. However, we show that increasing the length of DNA between the recombination sites and the enhancer and changing the sequence of the core nucleotides where strand exchange occurs increases the efficiency of the knotting reaction. The structure of the knots generated by different mutant substrates strongly supports a model involving a unique configuration of DNA strands at synapsis and DNA strand exchange mediated by rotation of one set of Hin subunits after DNA cleavage. Analysis of the stereostructure of the knots by electron microscopy of RecA-coated DNA molecules demonstrates that the direction of subunit rotation is exclusively clockwise. Because multiple subunit rotations generating knotted molecules do not occur efficiently when the enhancer is located in its native position, we suggest that the enhancer normally remains associated with the two recombination sites in the invertasome structure during strand exchange to limit strand rotation once it has been initiated. Under certain conditions, however, complex knots are formed that are probably the result of the premature release of the enhancer and multiple, unrestrained subunit exchanges.

[Key Words: Hin; Fis; recombinational enhancer; site-specific recombination; DNA topology]

Received March 15, 1991; revised version accepted June 18, 1991.

The Hin-mediated site-specific DNA recombination reaction is a well-defined system for investigating the widespread phenomenon of interaction between distantly located DNA sequences. In this system, proteinbound DNA sites, which can be located several kilobase pairs away from each other, assemble into a highly ordered reactive complex. This assembly requires longrange DNA looping and localized DNA bending, as well as homologous and heterologous protein-protein interactions (Johnson and Simon 1987; Johnson et al. 1987; Heichman and Johnson 1990|. To complete a successful reaction in this or any site-specific recombination reaction, the DNA strands and proteins must be aligned in a very precise configuration. Misalignment or instability of the complex during recombination would be expected to have serious or fatal consequences for the cell. There-

Present address: ${ }^{1}$ Department of Biochemistry, University of Wisconsin, Madison, Wisconsin 53706 USA.

${ }^{2}$ Corresponding author. fore, there must be a high degree of fidelity in this reaction.

The DNA inversion systems are distinguished by their requirement for an enhancer segment that functions to promote recombination at specialized sites (Johnson and Simon 1985). These recombination sites can be located from 50 to $>4000 \mathrm{bp}$ from the recombinational enhancer. In the case of the Hin recombination system of Salmonella, the enhancer is positioned within the 993bp invertible segment at $\sim 100$ bp from one of the two recombination sites. DNA inversion of this segment of the chromosome changes the orientation of a promoter that controls flagellin synthesis (Silverman and Simon 1980; Zieg and Simon 1980). Related inversion systems from bacteriophage $\mathrm{Mu}$ and $\mathrm{P1}$ have been described (Kamp et al. 1978; Iida et al. 1982) that function to regulate tail fiber expression.

The Hin-mediated DNA inversion reaction can be reconstituted in vitro with purified proteins and a supercoiled DNA substrate (Johnson et al. 1986). The reaction 
is very efficient; after only $10-15 \mathrm{~min}$ of incubation at $37^{\circ} \mathrm{C}$, an equilibrium state is reached where both orientations of the invertible segment are equally represented. The $21-\mathrm{kD}$ Hin protein binds as a dimer to each of the two 26-bp recombination sites and catalyzes a doublestrand cleavage at the central (core) dinucleotides of these sites to generate 2-bp 3'-OH overhangs (Glasgow et al. 1989; Johnson and Bruist 1989). Hin maintains the high energy phosphate bond by a covalent attachment to $5^{\prime}-\mathrm{PO}_{4}$ and subsequently catalyzes the resealing of DNA strands. A second protein, Fis, is also required for efficient inversion (Johnson et al. 1986; Koch and Kahmann 1986; Bruist et al. 1987). Fis binds to two sites on the 63-bp Hin recombinational enhancer sequence, both of which are required for enhancer activity. A mutation in either binding site destroys the function of the enhancer and results in a 100-fold decrease in recombination rates (Johnson and Simon 1985). The two recombination sites and the enhancer have been shown by electron microscopy to be associated in a nucleoprotein structure called an invertasome (Heichman and Johnson 1990). Both Hin and Fis are colocalized in this structure, which is believed to be the reaction intermediate where DNA strand exchange occurs (Fig. 1). The precise role of Fis and the enhancer in the recombination reaction is not well understood, although both are required for steps leading up to invertasome formation and DNA strand exchange. $\mathrm{HU}$ is a nonspecific DNA-binding protein that is required for efficient recombination when the enhancer is located close to a recombination site, presumably to facilitate DNA looping (Johnson et al. 1986).

Topological analysis of the products of several sitespecific recombination reactions has been an important tool in elucidating the mechanism of site synapsis and strand exchange (Wasserman and Cozzarelli 1986). For example, early work on knots generated in DNA during

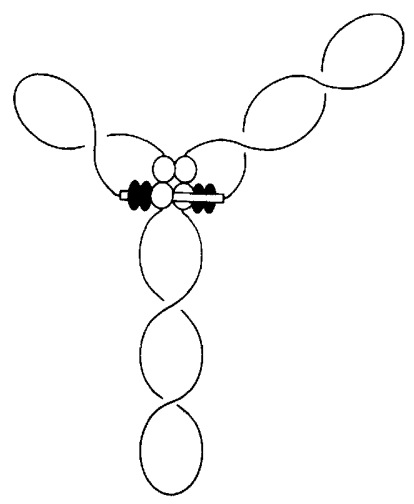

Figure 1. Structure of the Hin invertasome. A model for the structure of the Hin invertasome, which is the intermediate nucleoprotein structure in which DNA strand exchange takes place, is shown. The recombination sites bound by the Hin recombinase dimers (open spheres) are joined together at the recombinational enhancer segment, which is bound by two Fis dimers (solid ellipses). The precise locations of the proteins within this complex are not known. the $\lambda$ integration reaction demonstrated that the two recombination sites synapsed via random collision and that the DNA was wrapped around the attP site in a left-handed configuration (Pollock and Nash 1983; Griffith and Nash 1985; Spengler et al. 1985). A topological analysis of resolvase-mediated deletion has also been useful for understanding this recombination reaction. The discovery that the primary products of recombination in the resolvase system are singly interlinked catenanes, combined with the examination of rare knotted forms that have undergone multiple strand exchanges, has placed clear constraints on models describing the mechanism of synapsis and strand exchange (Krasnow and Cozzarelli 1983; Wasserman and Cozzarelli 1985; Wasserman et al. 1985; Stark et al. 1989).

In this paper we examine the knotting properties of the Hin-mediated DNA inversion reaction. Modest changes in the DNA substrate have made it possible to increase the efficiency of DNA knotting, which is normally a rare reaction. We find that different plasmid substrates produce unique populations of knotted products. The detailed analysis of the structure of the knots, the rate of their formation, and the conditions that favor their production have allowed us to determine the relative geometry of DNA strands within the invertasome and have provided strong evidence for a mechanism of strand exchange. These data have also provided information regarding the structure of the recombination complex during strand exchange and the role of the recombinational enhancer in synapsis and strand exchange.

\section{Results}

\section{Hin-promoted knotting of DNA}

Evidence for an efficient DNA knotting reaction by the Hin recombination system was first obtained with a plasmid substrate that contained nonidentical core sequences within the recombination sites. pRJ862 (Fig. 2) has one wild-type recombination site (hix $L-W T$ ) and one mutant site containing an $\mathrm{A} \rightarrow \mathrm{T}$ transversion within the core nucleotides (hix $L-A T$ ). This mutation prevents the formation of inversion products (Johnson and Simon 1985). pRJ862 also contains the enhancer segment beginning 694 bp from the center of the closest recombination site, in contrast to the native positioning in which the enhancer is $99 \mathrm{bp}$ from hixL. When pRJ862 is incubated under normal in vitro inversion conditions and electrophoresed in an agarose gel, a series of bands that migrate faster than the unreacted supercoiled molecules are observed (Fig. 3, lane 6). These knotted forms can be better resolved after nicking the DNA to remove supercoils (Fig. 3, lane 8). After a $30-\mathrm{min}$ reaction, $>80 \%$ of the pRJ862 DNA can be found in knotted forms of varying complexity.

The efficient production of knots in pRJ862 encouraged us to examine the knotting behavior of a plasmid that contains wild-type recombination sites and supports efficient inversion. pMS634 has the same structure 
A

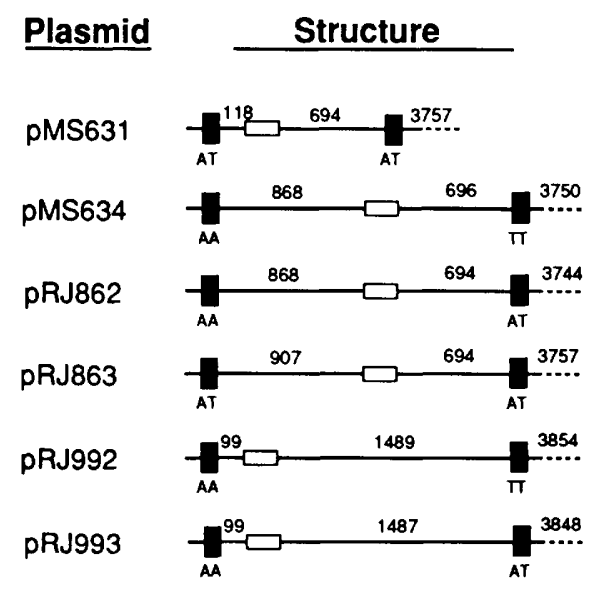

B

hixL-WT TTCTTGAAAACCAAGGTTTTTGATAA

hixL-AT TTCTTGAAAACCATGGTTTTTGATAA AAGAACTTTTGGTACCAAAAACTATT

Figure 2. Structure of the plasmid substrates used in this study. $(A)$ The solid vertical rectangles denote the recombination sites, with AA or TT indicating the wild-type core sequence of hix $L-W T$ and AT indicating the mutant core sequence of $h i x L-A T$. In all cases, the recombination sites are located in inverted orientation with respect to each other. The location of the 63-bp enhancer segment is denoted by the open rectangle. The number of base pairs between the center of the recombination sites and the boundaries of the enhancer are given, along with the length of the vector sequences (above the broken lines|, as measured between the center of the two recombination sites. $(B)$ Sequences of the wild-type hixL recombination site (hixL-WT) and a mutant hix $L$ recombination site (hix $L-A T)$. The bold nucleotides denote the core residues where DNA strand exchange takes place and where the only sequence difference between the two hix $L$ sites is located.

as pRJ862 except that both recombination sites are the wild-type hixL sequence (Fig. 2). This plasmid supports an efficient Hin-mediated topoisomerase reaction (Fig. 3, lane 2) that obscures visualization of any knotted forms. Upon nicking to remove the supercoils, however, a small but significant number of knots are detected (Fig. 3, lane 4). After a $30-$ min reaction, pMS634 accumulates $5-10 \%$ of its DNA in a knotted form.

These results show that the Hin recombination system can efficiently catalyze knotting of DNA. The knotting of DNA requires the presence of both recombination sites and the enhancer on a supercoiled plasmid (data not shown). The Fis protein, as well as HU when the enhancer is located at its native position, is also required in addition to the Hin recombinase. These features imply that the knotting reaction is intimately related to the
Hin-mediated inversion reaction because the two have the same requirements.

The presence of nonidentical core nucleotides within the recombination sites, combined with a relatively large spacing between the enhancer and the two recombination sites, strongly increases the efficiency of the knotting reaction (see below). Changes in reaction conditions can also have modest effects on the efficiency of knotting and structure of the products. For example, increasing the overall ionic strength or substituting $\mathrm{Mn}^{2+}$ for $\mathrm{Mg}^{2+}$ in the reaction results in modest changes in the knotting patterns and rates of knotting (data not shown). The absence of $\mathrm{Mg}^{2+}$ or any added divalent cation does not alter the Hin knotting reaction significantly, unlike results reported for the related Gin recombinase, which gives different rates and patterns of knotting with and without $\mathrm{Mg}^{2+}$ (Kanaar et al. 1990). The experiments described below are all performed under normal inversion conditions that include $\mathrm{Mg}^{2+}$.

\section{Structure of the DNA knots}

The number of DNA strand crossovers (nodes) in the various knotted forms was determined by gel electrophoresis and by electron microscopy. In Figure 4A, knotted products are electrophoresed in parallel with a ladder of knots produced by phage $\mathrm{T} 4$ topoisomerase (Liu et al. 1980). With pRJ862, the most prominent species are molecules containing an increasing number of nodes in multiples of three, with the more complex species increasing

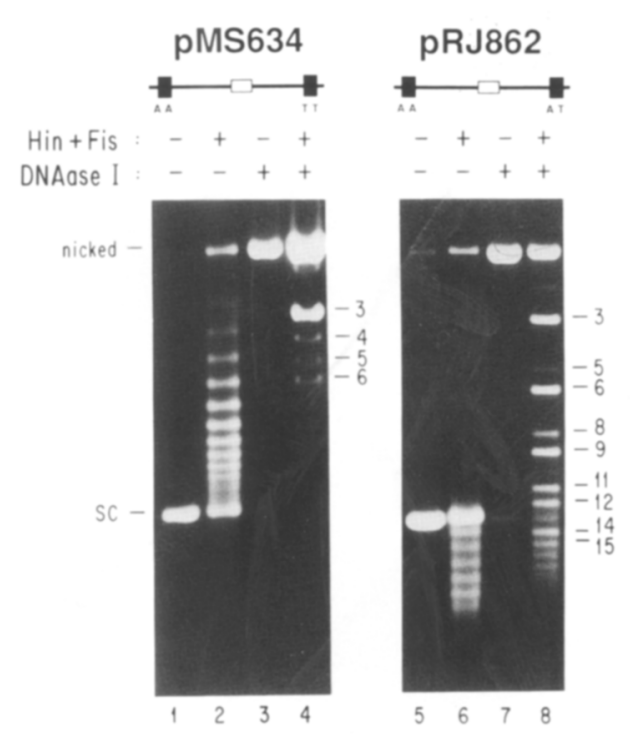

Figure 3. Hin-mediated topological changes in pMS634 and pRJ862. Agarose gel electrophoresis of pMS634 (lanes 1-4) and pRJ862 (lanes 5-8), which have the identical structure except that pRJ862 has one mutant hix L-AT site. The plasmids were reacted with $\mathrm{Hin}$, Fis, and $\mathrm{HU}$ for $30 \mathrm{~min}$, and a portion was nicked with DNase I, as indicated. The locations of the nicked and supercoiled $(\mathrm{SC})$ plasmid forms, as well as the number of nodes contained in the knotted species that have been nicked, are indicated. 


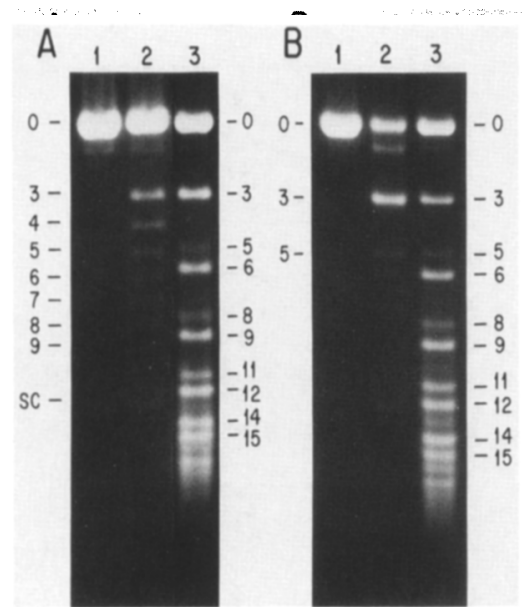

Figure 4. Structures of Hin-generated knots as determined by gel electrophoresis. (A) Lane 1 is the unreacted nicked plasmid. pRJ862 was reacted with T4 topoisomerase to generate a standard knotting ladder (lane 2), or with Hin, Fis, and HU proteins (lane 3), nicked with DNase I and electrophoresed in an agarose gel. The number of nodes contained in each of the T4 topoisomerase-generated species are indicated at left; the number of nodes contained in the major Hin-generated species are given at right $(B)$ Lane 1 is the unreacted nicked plasmid. pRJ862 was reacted with Hin, Fis, and HU under conditions that yield one round of recombination (lane 2; see Materials and methods) or under standard conditions for $30 \mathrm{~min}$ (lane 3 ), nicked with DNase I, and electrophoresed in an agarose gel. The number of nodes contained in each species seen after a single or multiple reactions is denoted at left and right, respectively.

with time (e.g., see Fig. 8A, below). Knotted DNA molecules containing up to 21 nodes have been resolved. In addition to the molecules containing multiples of 3 nodes, molecules with $5,8,11$, and 14 nodes are seen with increasing relative abundance. Under normal recombination conditions 4- and 7-noded molecules are not observed with pRJ862.

The assignment of the 3-, 5-, and 6-noded forms of pRJ862 has been confirmed by electron microscopy. Knotted DNA was eluted from gel slices, coated with RecA protein to thicken the DNA strands, and visualized after metal shadowing by electron microscopy (Krasnow et al. 1983; Griffith and Nash 1985). Figure 5 shows examples of molecules containing 3,5, and 6 nodes. The electron microscopy also allows us to determine the relative orientations of strand crossing and categorize the knots into various families; these results are summarized in Table 1. All 29 of the trefoils (3-noded knots) scored contained nodes of negative sign. All 10 of the 5-noded knots contained exclusively negative nodes, with most of these knots capable of assignment to the twist family of knots (Table 1; Rolfsen 1976). Most of the 6-noded knots can be definitively interpreted as containing two negative trefoils.

Gel electrophoresis of the knots generated in pMS634 indicates that the major species contains 3 nodes, with $4-, 5-$, and 6-noded forms being present in very low amounts (Fig. 3A; data not shown). Electron microscopy of 15 gel-purified trefoils of pMS634 (Fig. 5C; Table 1) demonstrates that each of these molecules contains exclusively negative nodes.

\section{Knots produced from one recombination complex}

A comparison of the complexity of the knots produced by pRJ862 with increasing time (e.g., see Fig. 8A, below) suggested that the more complex knots were the result of multiple reactions involving the reformation of an invertasome complex after a previous recombination event had been completed. The following experiment directly distinguishes between knots that are generated from assembly of one recombination complex and those that were generated from multiple reactions. pRJ862 was incubated for $15 \mathrm{~min}$ in reactions lacking $\mathrm{Mg}^{2+}$ and containing ethylene glycol. Under these conditions, stable recombination intermediates accumulate that yield cleaved invertible segment and vector DNA upon removal of protein (Johnson and Bruist 1989). These reaction intermediates were chased to recombinant products
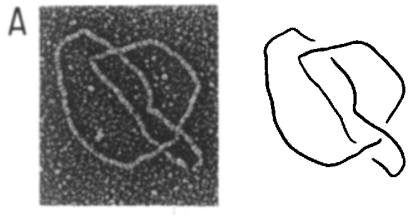

B
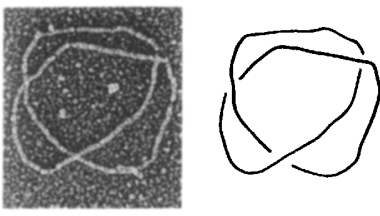

C
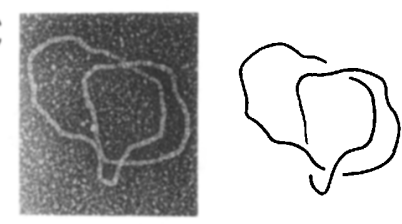

D
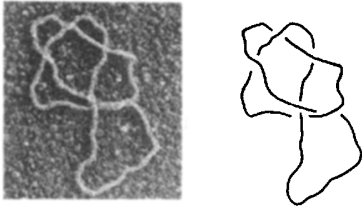

E

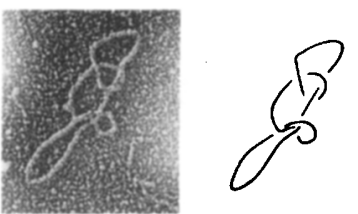

Figure 5. Electron microscopy of RecA-coated knots generated in PRJ862 and pMS634. All of the molecules contain exclusively nodes of the negative sign. $(A, B)$ Trefoils generated with pRJ862; $(C)$ a trefoil generated with $\mathrm{pMS634} ;(D)$ a 5-noded knot of pRJ862; $(E)$ a 6-noded knot of pRJ862. Beside each photograph is an interpretation of the knot structure. 
Table 1. Quantitation of RecA-coated knotted DNA molecules observed by electron microscopy

\begin{tabular}{lcccccc}
\hline & $\begin{array}{l}\text { 3-Noded } \\
\text { (all }-1\end{array}$ & $\begin{array}{l}\text { 3-Noded } \\
\text { (all }+)\end{array}$ & $\begin{array}{c}\text { 4-Noded } \\
(2+, 2-)\end{array}$ & $\begin{array}{c}\text { 5-Noded } \\
\text { (all }-)\end{array}$ & $\begin{array}{c}\text { 6-Noded } \\
\text { (all }-)\end{array}$ & $\begin{array}{c}\text { 6-Noded } \\
(2+, 4-)\end{array}$ \\
\hline pRJ862 & 29 & 0 & NP & 10 & 17 & ND \\
pMS634 & 15 & 0 & ND & ND & ND \\
pMS631 & 16 & 0 & 38 & 8 & 7 \\
\hline
\end{tabular}

RecA-coated DNA knots were photographed, and the sign and number of nodes were determined. Under each column heading, the sign of the nodes is indicated in parentheses. (NP) Not present. (ND) Not determined.

by incubating at $23^{\circ} \mathrm{C}$ for 1 min after diluting the ethylene glycol and adding $\mathrm{Mg}^{2+}$. Such recombinant products would be expected to have undergone only one round of recombination. As shown in Figure 4B, lane 2, only 3and 5-noded knots are formed from one round of recombination. Molecules reacted under standard conditions that contain 6 or more nodes (lane 3) must have undergone multiple reactions. Thus, molecules containing 6 and 8 nodes have most likely been generated from two independent reactions, molecules containing 9 and 11 nodes have most likely undergone 3 reactions, and so forth.

The same experiment was performed with pMS634 containing two hix $L-W T$ sites. As shown previously with a related substrate (Johnson and Bruist 1989), most of the molecules containing cleavages prior to dilution and $\mathrm{Mg}^{2+}$ addition were chased into inverted products that were unknotted. However, a few percent of the product molecules formed 3-noded knots and a trace formed 4- and 5-noded knots (data not shown). The very small number of 6-noded knots generated from long incubations with pMS634 (Fig. 3, lane 4) are most likely the result of two independent knotting reactions.

The results with pRJ862 and pMS634 strongly suggest that the knotted molecules are formed by multiple $180^{\circ}$ rotations of DNA strands within the recombination complex prior to ligation (see Figs. 6 and 7). When two wild-type recombination sites are utilized, the core nucleotides will be complementary at each $180^{\circ}$ rotation
(Fig. 6A) and the DNA strands can ligate efficiently after the first rotation without generating a knot. Plasmids such as pRJ862, which contain recombination sites with nucleotide differences in the core sequences, are unable to ligate after a single $180^{\circ}$ rotation due to noncomplementarity (Fig. 6B). An additional $180^{\circ}$ rotation will regenerate the parental primary structure and thus allow for ligation but will result in a 3-noded knot (Fig. 7). Another $180^{\circ}$ rotation will again leave the core bases unable to hydrogen-bond, demanding a fourth rotation to reseal the molecule into a 5-noded twist knot. Normally, $<1 \%$ of the pRJ862 molecules fail to ligate at this point and undergo further rotations. Thus, the inability of pRJ862 to ligate in the inverted orientation explains the finding that neither inversions nor 4- or 7-noded knots are observed.

\section{Suppression of knotting when the enhancer is close to a recombination site}

The efficiency of the knotting reaction is strongly influenced by the location of the enhancer relative to the recombination sites. The rate of the knotting reaction and the complexity of the knots are reduced dramatically when the enhancer is positioned close to one of the recombination sites. The effect of enhancer position can be illustrated by comparing the rate and pattern of knotting with pRJ862 versus pRJ993. Both plasmids have the hix $L-W T$ and hix $L-A T$ recombination sites separated by

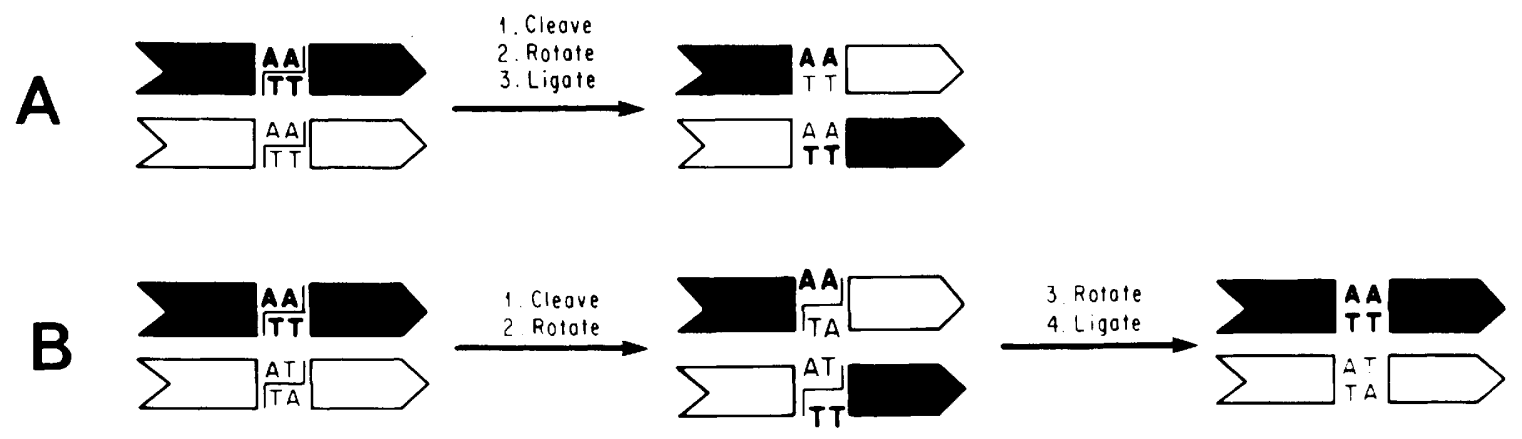

Figure 6. Schematic representation of synapsis and strand exchange of wild-type and mutant recombination sites. $(A)$ Two wild-type recombination sites (one solid and one open) are aligned together at synapsis. A staggered cleavage within the core nucleotides, followed by exchange of the half-sites and ligation as shown, results in inversion of the intervening DNA segment. $(B)$ One wild-type (solid structure) and one mutant (open structure) recombination site are aligned together at synapsis. DNA cleavage and exchange as in $A$ generates non-base-paired core sequences that cannot ligate efficiently. A second exchange restores base-pairing and thus allows for efficient ligation. The two exchanges restore the parental configuration of the DNA but generate a 3-noded knot. 
$\sim 1600$ bp, but pRJ993 has the enhancer at its native position 99 bp from hixL (see Fig. 2). The enhancer in pRJ862 is separated from the two recombination sites by 868 and 694 bp. Figure 8 shows that the initial rate and yield of knotted molecules after 20 min by pRJ993 is $\sim 10 \%$ of pRJ862. This suggests that topological constraints imposed by a close enhancer suppress the efficiency of knotting. The rate of inversion, however, between analogous plasmids containing two hix $L-W T$ sites (pMS634 and pRJ992; see Fig. 2) is identical (Fig. 9), indicating that the assembly of the invertasome and the first $180^{\circ}$ rotation is not inhibited when the enhancer is

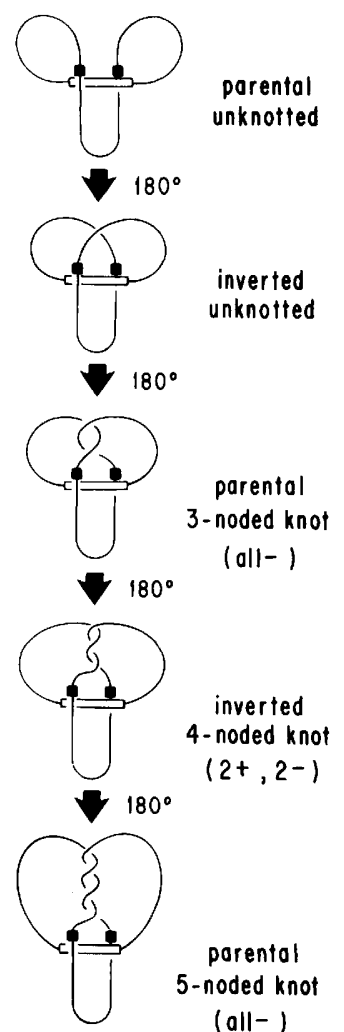

Figure 7. Model for the generation of knots that form during multiple rotations of DNA strands within a recombination complex. The DNA strands are assembled into the invertasome structure. Double-strand cleavages in both recombination sites, followed by a $180^{\circ}$ clockwise rotation of strands, generate an unknotted plasmid containing an inversion of the intervening DNA. A second $180^{\circ}$ rotation results in a 3-noded knot of negative sign with the intervening segment back into the parental configuration. A third $180^{\circ}$ rotation generates a knotted plasmid containing 4 nodes ( 2 positive and 2 negative) and an inversion. A fourth rotation generates a knotted plasmid containing 5 nodes (all negative) and no inversion. Further rotations of DNA within a recombination complex can occur rarely. For simplicity, the DNA is shown here as relaxed, and changes in supercoiling that accompany each rotation are not shown. A loss of four negative supercoils would be predicted to occur after the first $180^{\circ}$ rotation. Two or more $180^{\circ}$ rotations would be predicted to result in a net loss of two negative supercoils as compared with the starting structure.
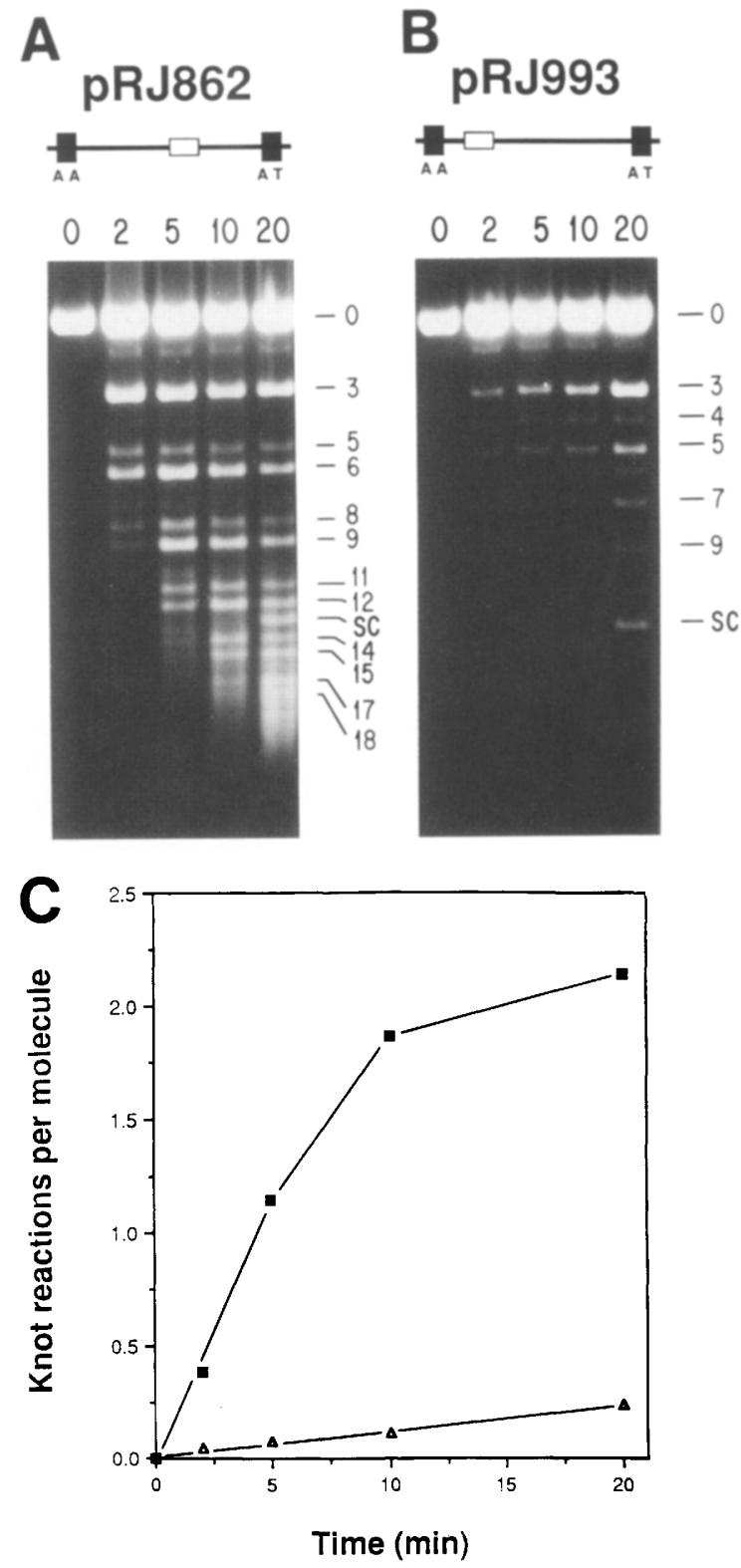

Figure 8. Effect of enhancer positioning on the rate of knotting. pRJ862 and pRJ993, which have very similar structures, except that the enhancer in pRJ993 is close to one of the recombination sites, were incubated with Hin, Fis, and HU for 0, 2, 5, 10 , and $20 \mathrm{~min}$. After nicking with DNase I, the plasmids were electrophoresed in an agarose gel $(A, B)$. The number of nodes contained in the knotted forms are indicated at right. (SC) The location of the supercoiled form that escaped nicking by the DNase. $(C)$ The number of knot reactions requiring independent assemblies of recombination complexes per substrate molecule is plotted as a function of time using the data obtained in $A$ (pRJ862, $\mathbf{a})$ and $B$ (pRJ993, $\mathbf{\Delta})$. The integration values from densitometry were adjusted for the minimum number of independent reactions that would be required to generate each species. For example, in the case of pRJ862, the values for 6- and 8-noded species were multiplied by two, as these species are most likely produced from two reactions; the 6-noded knot as the product of two reactions generating 3-noded knots; and the 8-noded knot as the product of one reaction producing one 3 - and one 5 -noded knot. 


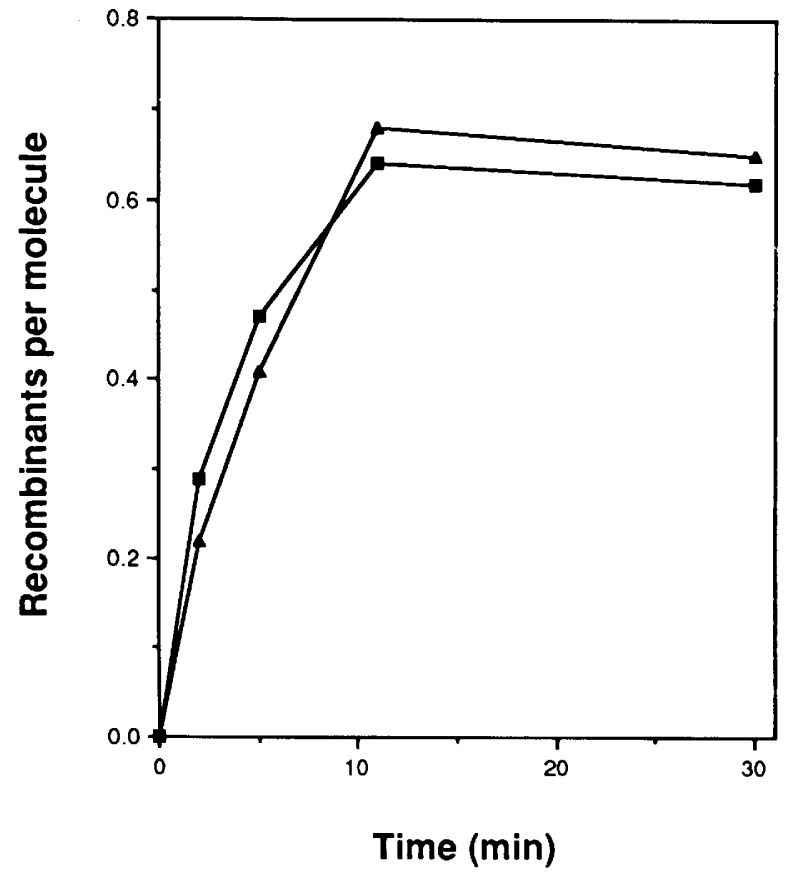

Figure 9. Effect of enhancer positioning on the rate of inversion. Inversion rates were measured for pMS634 ( $\mathbf{\square})$ and pRJ992 (A). These plasmids have very similar structures except that the enhancer in pRJ992 is close to one of the recombination sites. The results are plotted as inversions per molecule (Bruist and Simon 1984) as a function of time.

located 99 bp from a recombination site. Therefore, positioning of the enhancer at its native location only suppresses the ability to undergo two or more iterative rotations.

The pattern of knots generated in pRJ993 is markedly different than that seen with pRJ862. In the case of pRJ993, a repeating pattern with increases of 2 nodes is observed, generating molecules with 3,5, 7, and 9 nodes. These forms are most likely generated by an exclusively iterative process in which many rotations occur within a single recombination complex. Thus, the 7-noded species, which is not seen with pRJ862, is probably the result of six iterative $180^{\circ}$ rotations, and the 9-noded species is the result of eight iterative $180^{\circ}$ rotations. In addition, a detectable number of 4-noded knots that are not observed with pRJ862 are observed with pRJ993. These knots are probably generated after three rotations and ligation of mismatched core sequences, resulting in inverted molecules. Hin is able to catalyze $\sim 1-2 \%$ inversion after long incubations with pRJ993 even though the core sequences of the two recombination sites contain a 1-bp mismatch (data not shown). Inversion in pRJ862 has not been detected, even under conditions where onetenth of this rate could have been measured.

\section{Knotting properties of plasmids containing two mutant recombination sites}

We have also analyzed the knotting properties of substrates with both recombination sites containing identi- cal mutant core sequences. pMS631 and pRJ863 contain two hix $L-A T$ sites, with the enhancer located at different spacings (Fig. 2). As expected, these plasmids support efficient inversion as hydrogen bonding can occur after the first rotation (Johnson and Simon 1985). These plasmids, however, knot at an unexpectedly higher rate and generate more complex species than analogous plasmids containing wild-type recombination sites. As shown in Figure 10 for pMS631, $\leqslant 50 \%$ of the plasmids are in a knotted form after a 60-min incubation, with each species represented from 3 to $>9$ nodes. The rate of formation of the higher-order knots contrasts with those produced by $\mathrm{pRJ} 862$, in which there is a clear progression of increasing complexity with time. In the case of pMS631, highly complex knots are produced early in the reaction. Electron microscopy of RecA-coated knotted molecules was performed to confirm the identity of the pMS631 knot containing 3-6 nodes and to determine their stereostructure. Representative 3-, 4-, 5- and 6-noded molecules are shown in Figure 11, and the results are summarized in Table 1. All 16 of the trefoils contained nodes of the negative sign. The thirty-eight 4-noded knots contain two positive and two negative nodes and the eight 5 -noded molecules scored contain exclusively negative nodes. Most of the latter structures could be assigned to the twist family of knots. Gel electrophoresis resolves a doublet band at the 6-noded position after long reaction times (see Fig. 10), suggesting two forms of these molecules. Indeed, electron microscopy demonstrated that one population contains all negative nodes; a second population contains 4 negative and 2 positive nodes,

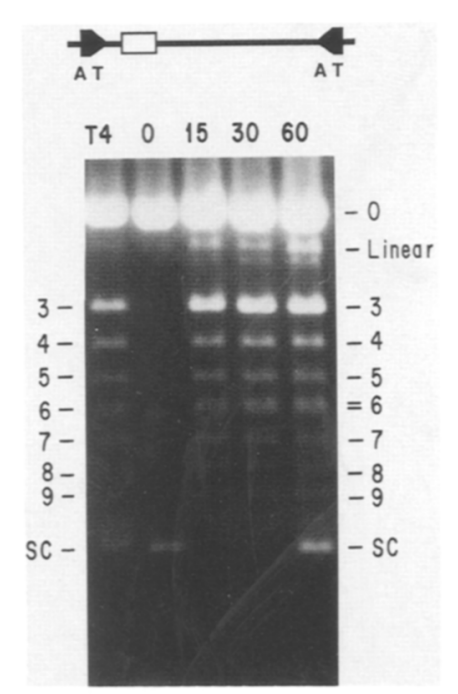

Figure 10. Hin-mediated knotting of pMS631. pMS63 I was reacted with Hin, Fis, and HU for 0, 15, 30, and 60 min as denoted, nicked with DNase I, and electrophoresed in an agarose gel. Lane T4 is a knotting ladder of pMS631 generated by T4 topoisomerase. The number of nodes in each species generated by $\mathrm{T} 4$ topoisomerase is indicated at left; the number of nodes in each species generated by Hin is indicated at right. (SC) The location of the supercoiled form that escaped nicking by the DNase. 
A
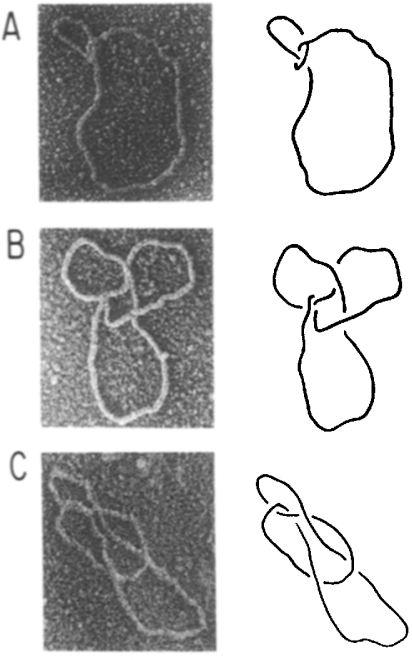

D

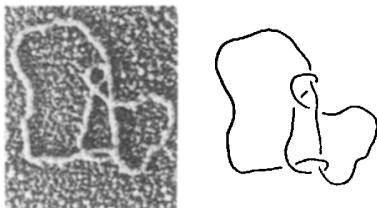

Figure 11. Electron microscopy of RecA-coated knots generated in pMS631. $(A)$ A trefoil containing all negative nodes; $|B|$ a 4-noded knot containing 2 negative and 2 positive nodes; $(C)$ a 5-noded knot with all negative nodes; $(D)$ a 6-noded knot with 4 negative and 2 positive nodes. Beside each photograph is an interpretation of the knot structure.

with the majority of these structures being most easily interpreted as twist knots (Fig. 11D, Table 1).

The formation of the highly complex knots with pMS631 is particularly surprising as the enhancer is located $118 \mathrm{bp}$ from a recombination site, which is similar to the wild-type spacing. Five-noded twist knots are most likely generated from four $180^{\circ}$ rotations within one recombination complex. Although the 6-noded knots of all negative sign are probably generated from two independent reactions, each generating 3-noded knots, the $4|-| 2|+| 6$-noded knots are most likely generated from an iterative mechanism involving five sequential rotations within a recombination complex. The rapid kinetics of formation of the knots containing $>6$ nodes is consistent with many of the knots being generated from an iterative mechanism, but direct analysis of their structure would be required to demonstrate this unequivocally. The significance of these highly iterative products with respect to the association of the enhancer with the recombination complex during the rotation of strands will be discussed below.

It is not known why plasmids containing two hix $L-A T$ sites knot more efficiently than similar substrates containing wild-type recombination sites. It has been observed, however, that a small number of these molecules remain unligated early in recombination reactions (data not shown). Such cleaved molecules are never seen with wild-type substrates and may reflect a slight inhibition of ligation with AT core nucleotides. Perhaps the precise alignment of strands in the recombination complex after strand rotation favors base-pairing and ligation of the wild-type (AA/TT) overhangs over the mutant (AT/TA) overhangs. This inhibition of ligation may provide an increased opportunity for further strand rotations.

\section{Discussion}

The Hin system is capable of efficiently knotting DNA under normal in vitro reaction conditions with the introduction of a few changes to the wild-type substrate. Plasmids with recombination sites containing different core nucleotides and larger spacing between these sites and the enhancer show initial rates of knotting that are very similar to inversion rates in the wild-type substrate. An analogous plasmid, with the same altered enhancer position but containing wild-type recombination sites, generates knots at $<5 \%$ of this rate but eventually accumulates $\sim 10 \%$ knotted molecules with long incubations. Different plasmids produce characteristic knotting patterns depending on their structure. For example, with pMS631, knots containing from 3 to $>9$ nodes are observed in decreasing abundance. In contrast, pRJ862 does not accumulate 4- and 7-noded knots and few 5- and 8-noded species, but many 3-, 6-, and 9-noded structures. We have shown that only the 3-and 5-noded species in pRJ862 are produced from one round of recombination and that the more complex knots, which increase with incubation time, must be the result of multiple reactions involving the reassembly of the recombination complex after the completion of the initial reaction.

\section{Mechanism of knotting}

The formation of unique patterns of knots in different substrates indicates that knotting must occur by means of a highly specific mechanism. Simple collision of recombination sites followed by strand exchange on a supercoiled molecule would not be expected to generate the knotting pattern and the types of knots we have observed. A mechanism that explains the formation of the patterns observed for each of the substrates is outlined in Figure 7. The DNA strands are assembled into the configuration depicted in the starting structure in which the two recombination sites bound by Hin dimers are joined together at the enhancer. Physical evidence for a nucleoprotein complex containing the enhancer and recombination sites has been obtained (Heichman and Johnson 1990). The DNA strands comprising the recombination sites cross the enhancer segment on opposite sides of the DNA helix (Fig. 1), consistent with the location of the Fis-binding sites on the enhancer (Johnson et al. 1986; Bruist et al. 1987) and a favorable configuration of branched supercoiled DNA (Kanaar et al. 1988). Strand exchange is initiated by double-strand cleavages at both recombination sites, leaving Hin covalently bound to the $5^{\prime}$ ends (Johnson and Bruist 1989). A $180^{\circ}$ rotation of one set of covalently bound subunits, as depicted schematically in Figure 12, inverts the intervening DNA segment. 


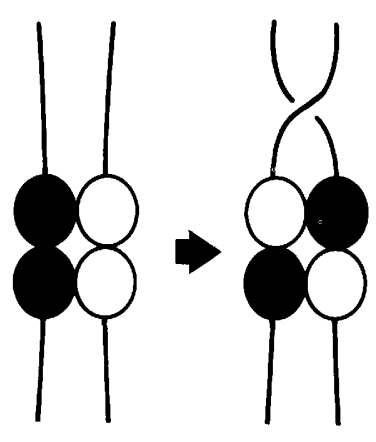

Figure 12. Model for recombination mediated by exchange of Hin subunits. Hin binds as a dimer $(\boldsymbol{\infty}$ or $(0)$ to each recombination site. Recombination proceeds by the clockwise rotation of one subunit from each dimer. The enhancer DNA segment and Fis proteins that are associated with the invertasome are not shown.

When base-pairing can occur, such as with two wild-type or two hix $L-A T$ sites, ligation will produce unknotted plasmids containing an inversion. Indeed, for pMS634, which has two hixL sites, $>95 \%$ of the reactions terminate after this step. However, a small number $<<\%$ for pMS634) fail to ligate and the subunits rotate an additional $180^{\circ}$ to yield trefoils that have the invertible segment oriented back into the parental configuration. Further subunit rotations followed by ligation will yield knotted molecules containing increasing numbers of nodes.

The high rate and unique pattern of knotting observed with pRJ862 is presumably a function of the inability to ligate in the inverted configuration. As diagramed in Figure 6, a single rotation of DNA strands places two noncomplementary core nucleotides together. Thus, an additional rotation is required to reseal the DNA strands, which generates a trefoil. Rotation in the opposite (counterclockwise/ direction would not be energetically favored on a negatively supercoiled molecule. Approximately $10 \%$ of the time the strands fail to ligate at this point and therefore must rotate two more $180^{\circ}$ turns to generate 5-noded knots of the twist family. The 5-noded knots characterized by electron microscopy provide evidence for this mechanism. An alternative mechanism for generating 5-noded twist knots involves two sequential reactions within a single invertasome complex, each completing two $180^{\circ}$ rotations. Four-noded knots are not produced because the core nucleotides would be in the recombinant configuration where they are unable to base-pair and ligate. The more complex knots result from multiple independent reactions. For example, two reactions, each generating 3-noded knots, yield molecules with 6 nodes; and two reactions, with one generating a 3-noded knot and the other generating a 5-noded knot, produce a molecule with 8 nodes. The stereostructure of the pRJ862 6-noded knots analyzed is consistent with their being formed from two sequential reactions. If the knots were the result of only one reaction with five $180^{\circ}$ rotations, they would be predicted to contain 2 pos- itive nodes and 4 negative nodes. In contrast, all of the pRJ862 6-noded knots contained only negative nodes. The plasmids observed that contain 21 nodes have most likely undergone seven independent reactions, with each reaction adding 3 additional nodes to the starting molecule.

\section{Direction of DNA strand rotation during recombination}

Specific predictions can be made regarding the sign of the nodes for each species, depending on whether DNA strand rotation proceeds in a clockwise or counterclockwise direction. In a clockwise rotation, 3- and 5-noded molecules will contain all nodes of negative sign, whereas a counterclockwise rotation will generate nodes of positive sign. Because the sixty 3 -noded and eighteen 5 -noded molecules observed in reactions with pRJ862, pMS631, and pMS634 contained exclusively negative nodes, the rotation of DNA strands must be in the clockwise direction, as shown in Figures 7 and 11. This is the direction that would be energetically favored when the invertasome is assembled on a negatively supercoiled DNA molecule, suggesting that the supercoiling may provide torque to drive the rotation of strands. The finding that recombination catalyzed by Fis-independent mutants of the Gin recombinase can occur in the absence of DNA supercoiling (Klippel et al. 1988) demonstrates that, at least under certain conditions, supercoiling is not required for subunit rotation in these systems. Constraints imposed by the structure of the invertasome may also impose directionality on the rotation, as has been proposed for Tn3 resolvase synaptic complex (Dröge and Cozzarelli 1989).

\section{Possible function of the recombinational enhancer in initiating subunit rotation}

We have shown previously that two types of nucleoprotein structures are generated in reactions containing the Hin and Fis proteins: invertasomes and paired hix complexes (Heichman and Johnson 1990). This work and previous studies on both the Hin (Heichman and Johnson 1990) and Gin (Kanaar et al. 1988) inversion systems strongly suggest that inversion initiates in the invertasome structure. The paired hix structures may be precursors of invertasomes that, in the absence of an enhancer, do not undergo strand exchange. There are at least two models that can account for the requirement of the enhancer to initiate recombination. The first is that the recombination sites and/or Hin protomers in the paired hix complex are not correctly aligned into a productive synaptic complex. The presence of the enhancer may serve to adjust the components into a configuration that is competent to recombine (Johnson and Simon 1987). However, there is evidence suggesting that the paired hix complex may be in a "synaptic" conformation. This includes the ability of Hin to catalyze doublestrand cleavages within the core nucleotides under certain reaction conditions without Fis or a functional 
enhancer (Johnson and Bruist 1989). In addition, single amino acid substitutions in the highly related recombinases Gin and Cin enable these enzymes to promote recombination in the absence of Fis or an enhancer (Haffter and Bickle 1988; Klippel et al. 1988). It seems reasonable that the wild-type recombinase can associate the recombination sites into a bona fide synaptic structure which, without the enhancer, is incapable of completing the recombination reaction.

A second model proposes that the paired hix structures are aligned correctly into a synaptic complex but cannot undergo strand exchange. The association of the enhancer provides the "activation energy" that enables the Hin protomers to perform DNA cleavage or subunit rotation or some other rate-limiting step after synapsis. Because paired hix structures can support a modest DNA cleavage reaction (Johnson and Bruist 1989), an attractive possibility is that subunit rotation may be the step limiting recombination in the paired hix complex. To initiate subunit rotation after cleavage of the DNA, the Hin dimerization contacts must be released while the contacts with the Hin protomers on the synapsed site must be maintained. This step may require some conformational changes or reorganization of the recombination complex that is induced by the association of the enhancer. As described below, the knotting behavior of pMS631 indicates that the enhancer is required only to initiate strand exchange and, thus, by this model, initiate subunit rotation.

\section{Association of the recombinational enhancer with the recombination complex during strand exchange}

Knotting experiments with substrates containing variable spacing between hix sites and the enhancer can address whether the enhancer remains physically associated with the recombination complex after strand exchange has been initiated. One could imagine three distinct scenarios, which are schematically represented in Figure 13: The enhancer remains associated with the recombination complex during strand exchange $(\mathrm{A})$; the enhancer is required to assemble a functional synaptic complex and/or initiate strand exchange and is then released (B); or the enhancer is released after strand exchange is initiated but must reassociate to initiate each $180^{\circ}$ rotation $(\mathrm{C})$. The first two possibilities can be distinguished by comparing the rates of knotting of substrates containing a short or long DNA segment between the enhancer and the closest recombination site (pRJ993 and pRJ862, respectively). If the enhancer remains associated with the recombination sites during strand exchange (pathway A), a short DNA segment would be expected to constrain the number of rotations possible. On the other hand, if the enhancer is released, either by the release of the DNA segment from the complex (Fig. 12) or by the threading of the DNA through the complex, the torsional constraint will be reduced, resulting in a much greater freedom of DNA strand rotation. It was found that reactions involving multiple strand rotations were dramatically inhibited when the enhancer was located

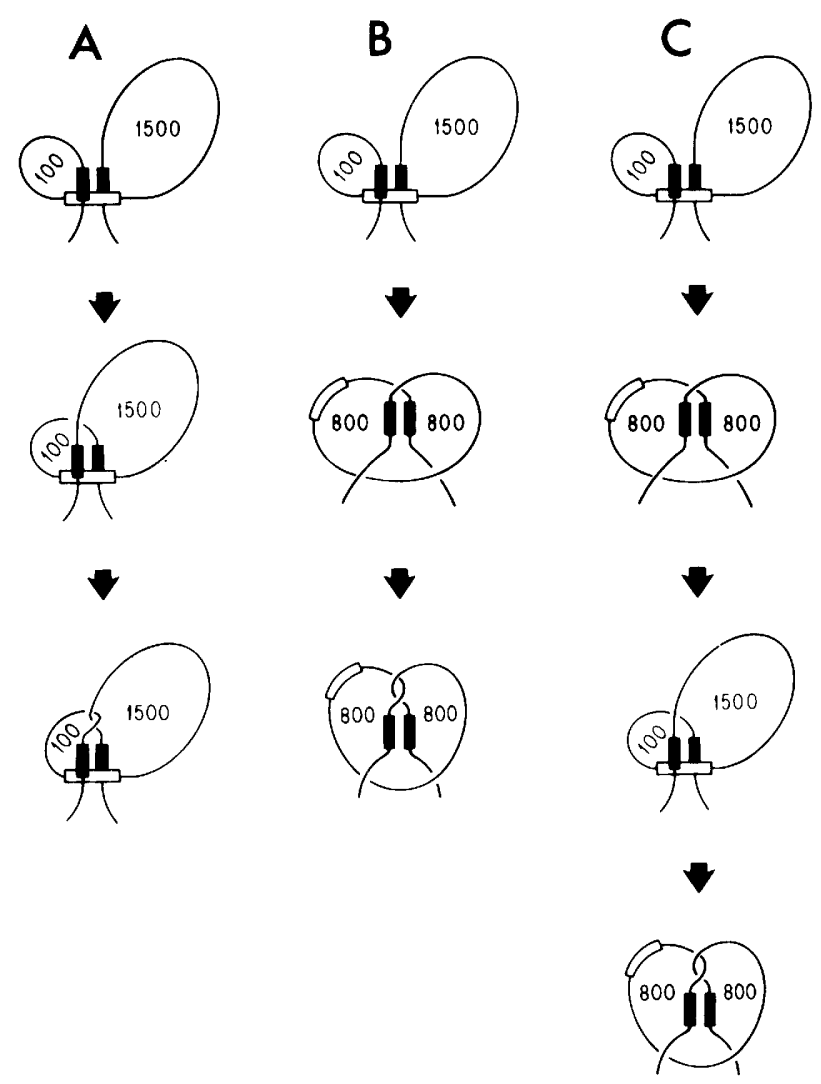

Figure 13. Retention or release of the enhancer during recombination. In pathway $A$, the enhancer remains associated with the recombination complex during two DNA strand rotations. In pathway $B$, the enhancer is released prior to or during the first strand rotation. Subsequent rotations occur without reassociation of the enhancer with the recombination sites. In pathway $C$, the enhancer is released prior to or during the first strand rotation as in pathway $B$. A subsequent rotation of DNA strands requires reassociation of the enhancer with the synapsed recombination sites.

close to a recombination site, even though the rate of inversion was the same. Therefore, the enhancer must normally remain associated with the recombination sites during strand exchange or reassociate for each rotation.

Two further observations are consistent with this conclusion. Reactions on substrates containing nonidentical cores result in a significant number of molecules containing double-strand cleavages at both recombination sites after the addition of protein denaturants (Johnson and Bruist 1989). Cleaved molecules are produced in pRJ862 at $10-20 \%$ of the amounts produced in pRJ993 (R.C. Johnson, unpubl.|, presumably because the subunits can readily undergo a second rotation to ligate the ends in pRJ862. A second observation is that a very low number of molecules containing inversions $(1-2 \%$ after $30 \mathrm{~min}$ ) and 4-noded knots are obtained with pRJ993, but neither are detected in pRJ862. Again, a short spacing between the enhancer and recombination site may constrain the DNA strands in the recombinant configura- 
tion after a single $180^{\circ}$ rotation, resulting in a greater chance for ligation between non-Watson-Crick-bonded base pairs.

Pathway $C$, in which the enhancer must reassociate to initiate every subunit rotation, cannot be distinguished definitively from pathway $\mathrm{A}$, as both would be expected to have similar topological properties. However, the knotting behavior of pMS631 suggests that the enhancer does not have to be physically associated with the recombination sites for each rotation once strand exchange has been initiated. The low rate of knotting with pRJ993 implies that even two rotations are inhibited at the wildtype spacing of the enhancer from hixL. On the other hand, reactions with pMS631, which has a similar spacing to pRJ993, generate significant numbers of molecules that have undergone at least five rotations within a recombination complex. For this to have occurred, the enhancer most likely has been permanently released. It is not clear why the enhancer is released from pMS631 more efficiently than from other substrates, but we note that a small number of highly iterative products are produced in reactions with pRJ993 where the enhancer is also located close to a recombination site. Perhaps the torsional stress generated by conditions that promote multiple rotations (such as mutant core sequences) causes the release of the enhancer when it is located close to a recombination site. This release of the enhancer then leads to swiveling of the Hin subunits.

The ability of a recombination complex to support multiple rotations of one set of subunits has profound implications for the nature of the complex. If there is such freedom of movement, one wonders how the complex remains efficiently associated such that broken molecules occur only very rarely, even when the complex is forced to undergo multiple rotations to be religated. One could imagine a ratchet-like mechanism for rotation where the subunits are temporarily fixed after each rotation to allow for ligation whenever possible. Thus, when the core nucleotides are complementary, as with the wild-type substrate, rotation is usually limited to one turn. In the case of mismatched core sequences, ligation is usually achieved after two rotations. The finding that highly complex knots are generated when the enhancer is released may indicate that the enhancer might also play some role in the ratcheting process, perhaps helping to fix the subunits after each rotation. The loss of the enhancer then leads to a greater degree of swiveling.

\section{Relationship to the Gin-mediated inversion reaction}

Similar synaptic intermediate structures and reaction mechanisms have been proposed for the Gin inversion reaction on the basis of topological analysis (Kanaar et al. 1988,1990 ). Gin-mediated inversion has been shown to be accompanied by a linking number change in the DNA of +4 (Kahmann et al. 1987; Kanaar et al. 1988); and under certain reaction conditions, Gin can knot DNA efficiently (Kanaar et al. 1990). In contrast to the Hin reaction with the wild-type substrate, the structure of the Gin-promoted knots indicates that the DNA strands have often undergone many rotations in substrates where the enhancer is located close to a recombination site. This has led to the proposal by Kanaar et al. (1990) that the enhancer in the Gin reaction is normally released early in the reaction. We believe that the enhancer normally remains associated with the Hin recombination complex. Under the rare conditions in which it is released, multiple subunit exchanges can occur to generate very complex knots from one initiating event.

\section{Materials and methods}

Enzymes and reagents

Hin was purified as described from Escherichia coli fis mutant cells (Johnson et al. 1986). Fis was purified from an overproducing E. coli strain by a modification of the procedure in Johnson et al. (1986), and HU was purified as described (Johnson et al. 1986); some of the experiments were performed with HU protein isolated from fis hip cells. T4 DNA topoisomerase was a generous gift from K. Kreuzer (Duke University). DNase I was purchased from Bethesda Research Laboratories, and DNA restriction enzymes were from Boehringer Mannheim Biochemicals or New England Biolabs.

\section{Recombination reactions}

Standard in vitro reactions for inversion or knotting were performed as follows: $0.1-0.2$ pmoles of plasmid DNA was incubated at $37^{\circ} \mathrm{C}$ with $50-70 \mathrm{ng}$ of Hin, $25 \mathrm{ng}$ of Fis, and $100 \mathrm{ng}$ of HU per 0.1 pmole of substrate DNA in a $25-\mu 1$ volume containing $20 \mathrm{~mm}$ Tris- $\mathrm{HCl}$ (pH 7.5), $100 \mathrm{~mm} \mathrm{NaCl}, 10 \mathrm{~mm} \mathrm{MgCl}_{2}, 1$ $\mathrm{mM}$ dithiothreitol, and $200 \mu \mathrm{g} / \mathrm{ml}$ of polycytidylic acid (Pharmacia). Reactions were terminated by heating to $70^{\circ} \mathrm{C}$ for 10 min or by the addition of $2 \mu \mathrm{l}$ of $1 \%$ diethylpyrocarbonate (Sigma). Reactions in which recombination was limited to one round were performed as described in Johnson and Bruist (1989).

Plasmids were nicked by incubation with $2.4 \mu \mathrm{g} / \mathrm{ml}$ of DNase I in the presence of $300 \mu \mathrm{g} / \mathrm{ml}$ of ethidium bromide for $30 \mathrm{~min}$ at $23^{\circ} \mathrm{C}$. The DNA was extracted with phenol/chloroform $(1: 1)$ prior to gel electrophoresis. Gel electrophoresis was performed in $0.85 \%$ agarose gels for $1.5-2$ days at $0.5 \mathrm{~V} / \mathrm{cm}$ in TPE buffer (10.8 grams of Tris base, $1.51 \mathrm{ml}$ of $85 \%$ phosphoric acid, and 0.74 grams of $\mathrm{Na}_{2}$ EDTA $/ 2 \mathrm{H}_{2} \mathrm{O}$ per liter). The DNA was visualized by staining with ethidium bromide. Quantitation of knotted species was performed by scanning Polaroid negatives with an LKB Ultroscan model 2202 laser densitometer, and the peak values were integrated with the LKB 2190 version 5.2 GelScan software package. A ladder of knots generated by T4 topoisomerase was produced by incubating the plasmid DNA with $0.7 \mu \mathrm{g}$ of the enzyme for $10 \mathrm{~min}$ at $30^{\circ} \mathrm{C}$ in a $25-\mu \mathrm{l}$ reaction containing $20 \mathrm{~mm}$ Tris-acetate $(\mathrm{pH} 7.9), 10 \mathrm{~mm}$ magnesium acetate, $60 \mathrm{~mm}$ potassium glutamate, $1 \mathrm{~mm}$ dithiothreitol, and 50 $\mu \mathrm{g} / \mathrm{ml}$ of bovine serum albumin. The plasmid was nicked with DNase I as described above prior to gel electrophoresis.

\section{Plasmids}

The relevant structures of the plasmids used in this study are given in Figure 2. All of the plasmids are derivatives of pBR322 and contain one hix $L-W T$ (or hix $L-A T$ ) at the EcoRI site and one hix $L-W T$ (or hix $L-A T$ ) at the Sall site in inverted orientation. The construction of pMS631 and pMS634 has been described previously (Johnson and Simon 1985). pRJ862 was con- 
structed from a ligation of the HindIII-PvuI DNA fragment from pMS634 that contains hixL-WT and the enhancer with the HindIII-PvuI fragment from pMS614 (Johnson and Simon 1985) that contains pBR322 ori and hixL-AT. A 789-bp HindII fragment containing the lacPT42 mutation (Reznikoff et al. 1982) was added to three plasmids to alter the spacing between a recombination site and the enhancer. pMS863 has the 789-bp fragment inserted into the unique SmaI site of pMS631. pRJ992 and pRJ993 have the 789-bp fragment inserted into the filled-in HindIII site of pMS551 and pMS614, respectively (Johnson and Simon 1985).

\section{Electron microscopy of DNA knots}

Knotted DNA molecules were isolated from slabs of agarose and purified according to the Geneclean (Bio101) protocol. The molecules were coated with RecA protein under the following conditions: $\sim 65 \mathrm{ng}$ of DNA was boiled for 2-3 min to denature the DNA strands and was then placed on ice immediately. The denatured DNA was incubated at $37^{\circ} \mathrm{C}$ for $15 \mathrm{~min}$ in a $30-\mu \mathrm{l}$ volume with $2.1 \mu \mathrm{g}$ and $0.18 \mu \mathrm{g}$ of RecA and SSB proteins, respectively (U.S. Biochemical) in a buffer containing $25 \mathrm{~mm}$ triethanolamine $(\mathrm{pH} 7.5), 10 \mathrm{~mm}$ sodium chloride, $5 \mathrm{~mm}$ magnesium acetate, and $0.6 \mathrm{~mm}$ ATP $\gamma \mathrm{S}$. Samples were then adsorbed onto carbon-coated 400-mesh grids that had been treated with Alcian blue, negatively stained with uranyl acetate, and rotary-shadowed with tungsten (Heichman and Johnson 1990). Molecules were observed and photographed with a JEOL JEM 1200 EX electron microscope. The sign of the nodes was designated according to the convention described in Wasserman and Cozzarelli (1986).

\section{Acknowledgments}

We thank Ken Kreuzer for a gift of T4 topoisomerase and Jack Griffith for useful advice on RecA coating of DNA. We are also grateful to the members of this laboratory for their useful comments on the manuscript. This work was supported by grant GM38509 from the National Institutes of Health and the Searle Scholars Program/The Chicago Community Trust. K.A.H. was supported in part by a U.S. Public Health Service National Institutional Research Service Award (CA-09056) and a Dr. Ursula Mandel Scholarship.

The publication costs of this article were defrayed in part by payment of page charges. This article must therefore be hereby marked "advertisement" in accordance with 18 USC section 1734 solely to indicate this fact.

\section{References}

Bruist, M.F. and M.I. Simon. 1984. Phase variation and the Hin protein: In vivo activity measurements, protein overproduction and purification. J. Bacteriol. 159: 71-79.

Bruist, M.F., A.C. Glasgow, R.C. Johnson, and M.I. Simon. 1987. Fis binding to the recombinational enhancer of the Hin DNA inversion system. Genes \& Dev. 1: 762-772.

Dröge, P. and N.R. Cozzarelli. 1989. Recombination of knotted substrates by Tn3 resolvase. Proc. Natl. Acad. Sci. 86: 60626066.

Glasgow, A.C., M.F. Bruist, and M.I. Simon. 1989. DNA binding of the Hin recombinase. J. Biol. Chem. 264: 10072-10082.

Griffith, J.D. and H.A. Nash. 1985. Genetic rearrangement of DNA induces knots with a unique topology: Implications for the mechanism of synapsis and crossing-over. Proc. Natl. Acad. Sci. 82: 3124-3128.
Haffter, P. and T.A. Bickle. 1988. Enhancer-independent mutants of the Cin recombinase have a relaxed topological specificity. EMBO I. 7: 3991-3996.

Heichman, K.A. and R.C. Johnson. 1990. The Hin invertasome: Protein-mediated joining of distant recombination sites at the enhancer. Science 249: 511-517.

Iida, S., J. Meyer, K. Kennedy, and W. Arber. 1982. A site-specific conservative recombination system carried by bacteriophage P1. EMBO T. 1: 1445-1453.

Johnson, R.C. and M.F. Bruist. 1989. Intermediates in Hin-mediated DNA inversion: A role for Fis and the recombinational enhancer in the strand exchange reaction. $E M B O J$. 8: $1581-1590$.

Johnson, R.C. and M.I. Simon. 1985. Hin-mediated site-specific recombination requires two 26 bp recombination sites and a 60 bp recombinational enhancer. Cell 41: 781-789.

. 1987. Enhancers of site-specific recombination in bacteria. Trends Genet. 3: 262-267.

Johnson, R.C., M.F. Bruist, and M.I. Simon. 1986. Host protein requirements for in vitro site-specific DNA inversion. Cell 46: $531-539$.

Johnson, R.C., A.C. Glasgow, and M.I. Simon. 1987. Spatial relationship of the Fis binding sites for Hin recombinational enhancer activity. Nature 329: 462-465.

Kahmann, R., G. Mertens, A. Klippel, B. Brauer, F. Rudt, and C. Koch. 1987. The mechanism of $\mathrm{G}$ inversion. In DNA replication and recombination (ed. R. McMacken and T.J. Kelly), pp. 681-690. Alan R. Liss, New York.

Kamp, P., R. Kahmann, D. Zipser, T.R. Broker, and L.T. Chow. 1978. Inversion of the G DNA segment of phage Mu controls phage infectivity. Nature 271: 577-580.

Kanaar, R., P. van de Putte, and N.R. Cozzarelli. 1988. Ginmediated DNA inversion: Product structure and the mechanism of strand exchange. Proc. Natl. Acad. Sci. 85: 752756.

Kanaar, R., A. Klippel, E. Shekhtman, J.M. Dungan, R. Kahmann, and N.R. Cozzarelli. 1990. Processive recombination by the phage $\mathrm{Mu}$ Gin system: Implications for the mechanisms of DNA strand exchange, DNA site alignment, and enhancer action. Cell 62: 353-366.

Klippel, A., K. Cloppenborg, and R. Kahmann. 1988. Isolation and characterization of unusual gin mutants. EMBO $\mathrm{I}$. 7: 3983-3989.

Koch, C. and R. Kahmann. 1986. Purification and properties of the Escherichia coli host factor required for inversion of the $\mathrm{G}$ segment in bacteriophage $\mathrm{Mu}$. J. Biol. Chem. 261: 1567315678.

Krasnow, M.A. and N.R. Cozzarelli. 1983. Site-specific relaxation and recombination by the Tn 3 resolvase: Recognition of the DNA path between oriented res sites. Cell 32: 13131324.

Krasnow, M.A., A. Stasiak, S.J. Spengler, F. Dean, T. Koller, and N.R. Cozzarelli. 1983. Determination of the absolute handedness of knots and catenanes of DNA. Nature 304: 559-560.

Liu, L.F., C.-C. Liu, and B.M. Alberts. 1980. Type II DNA topoisomerases: Enzymes that can unknot a topologically knotted DNA molecule via a reversible double-strand break. Cell 19: 697-707.

Pollock, T.J. and H.A. Nash. 1983. Knotting of DNA caused by a genetic rearrangement. J. Mol. Biol. 170: 1-18.

Reznikoff, W.S., L.E. Maquat, L.E. Munson, R.C. Johnson, and W. Mandecki. 1982. The lac promoter: Analysis of structural signals for transcription initiation and identification of a new sequence specific event. In Promoters: Structure and function (ed. R.L. Rodriguez and M.J. Chamberlin), pp. 80- 
95. Praeger Publishers, New York.

Rolfsen, D. 1976. Knots and links, p. 391. Publish or Perish, Inc., Berkeley, California.

Silverman, M. and M. Simon. 1980. Phase variation: Genetic analysis of switching mutants. Cell 19: 845-854.

Spengler, S.J., A. Stasiak, and N.R. Cozzarelli. 1985. The stereostructure of knots and catenanes produced by phage $\lambda$ integrative recombination: Implications for mechanism and DNA structure. Cell 42: 325-334.

Stark, W.M., D.J. Sherratt, and M.R. Boocock. 1989. Site-specific recombination by $\mathrm{Tn} 3$ resolvase: Topological changes in the forward and reverse reactions. Cell 58: 779-790.

Wasserman, S.A. and N.R. Cozzarelli. 1985. Determination of the stereostructure of the product of $\operatorname{Tn} 3$ resolvase by a general method. Proc. Natl. Acad. Sci. 82: 1079-1083.

1986. Biochemical topology: Applications to DNA recombination and replication. Science 232: 951-960.

Wasserman, S.A., J. Dungan, and N.R. Cozzarelli. 1985. Discovery of a predicted DNA knot substantiates a model for sitespecific recombination. Science 229: 171-174.

Zieg, J. and M. Simon. 1980. Analysis of the nucleotide sequence of an invertible controlling element. Proc. Natl. Acad. Sci. 77: 4196-4200. 


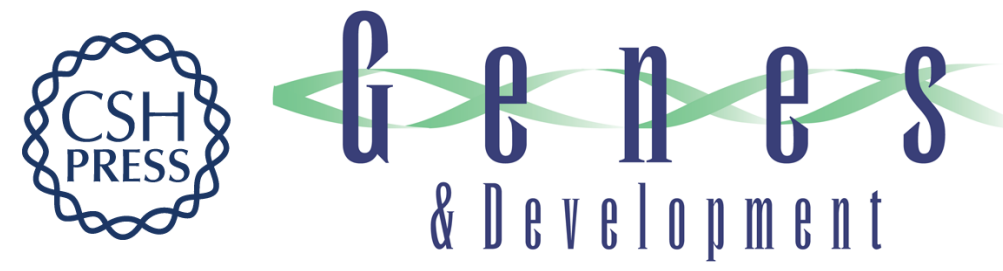

\section{Configuration of DNA strands and mechanism of strand exchange in the Hin invertasome as revealed by analysis of recombinant knots.}

K A Heichman, I P Moskowitz and R C Johnson

Genes Dev. 1991, 5:

Access the most recent version at doi:10.1101/gad.5.9.1622

References This article cites 29 articles, 12 of which can be accessed free at: http://genesdev.cshlp.org/content/5/9/1622.full.htmI\#ref-list-1

License

Email Alerting

Receive free email alerts when new articles cite this article - sign up in the box at the top Service right corner of the article or click here.

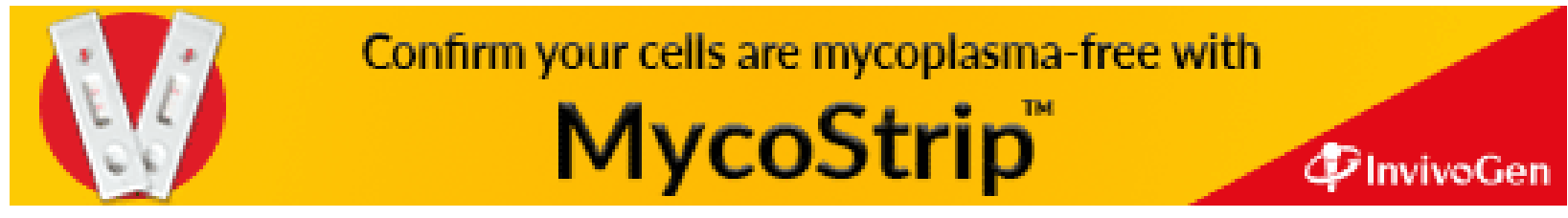

